\title{
CHROMATIC POLYNOMIALS OF MIXED HYPERCYCLES
}

\author{
Julian A. Allagan And David Slutzky \\ Department of Mathematics \\ University of North Georgia \\ Watkinsville, Georgia, USA \\ e-mail: julian.allagan@ung.edu \\ david.slutzky@ung.edu
}

\begin{abstract}
We color the vertices of each of the edges of a $\mathcal{C}$-hypergraph (or cohypergraph) in such a way that at least two vertices receive the same color and in every proper coloring of a $\mathcal{B}$-hypergraph (or bihypergraph), we forbid the cases when the vertices of any of its edges are colored with the same color (monochromatic) or when they are all colored with distinct colors (rainbow). In this paper, we determined explicit formulae for the chromatic polynomials of $\mathcal{C}$-hypercycles and $\mathcal{B}$-hypercycles.
\end{abstract}

Keywords: hypercycle, mixed hypergraph, chromatic polynomial.

2010 Mathematics Subject Classification: 05C15, 05C30.

\section{REFERENCES}

[1] J.A. Allagan, The chromatic polynomials of some linear uniform hypergraphs, Congr. Numer. 187 (2007) 156-160.

[2] C. Berge, Graphs and Hypergraphs (North-Holland, Amsterdam, 1973).

[3] A.A. Bhatti, S.A. Bokhary and I. Tomescu, On the chromaticity of multi-bridge hypergraphs, Graphs Combin. 25 (2009) 145-152.

doi:10.1007/s00373-008-0831-7

[4] M. Borowiecki and E. Eazuka, On chromaticity of hypergraphs, Discrete Math. 307 (2007) 1418-1429. doi:10.1016/j.disc.2005.11.079

[5] M. Borowiecki and E. Łazuka, Chromatic polynomials of hypergraphs, Discuss. Math. Graph Theory 20 (2000) 293-301.

doi:10.7151/dmgt.1128 
[6] F.M. Dong, K.M. Koh and K.L. Teo, Chromatic Polynomials and Chromaticity of Graphs (Company Singapore: World Scientific Publishing., 2005).

[7] G. Lo Faro, L. Milazzo and A. Tripodi, On the upper and lower chromatic number of BSQSS(16), Electron. J. Combin. 8 (2001)\#R6.

[8] M. Gionfriddo, L. Milazzo, A. Rosa and V. Voloshin, Bicolouring Steiner systems $S(2,4, v)$, Discrete Math. 283 (2004) 249-253. doi:10.1016/j.disc.2003.11.016

[9] D. Král', J. Kratochvil and H. Voss, Mixed hypercacti, Discrete Math. 286 (2004) 99-113. doi:10.1016/j.disc.2003.11.051

[10] T. Jiang, D. Mubayi, Zs. Tuza, V. Voloshin and D.B. West, The chromatic spectrum of mixed hypergraphs, Graphs Combin. 18 (2002) 309-318. doi:10.1007/s003730200023

[11] L. Milazzo and Zs. Tuza, Upper chromatic number of Steiner triple and quadruple systems, Discrete Math. 174 (1997) 247-259. doi:10.1016/S0012-365X(97)80332-9

[12] L. Milazzo and Zs. Tuza, Strict colouring for classes of Steiner triple systems, Discrete Math. 182 (1998) 233-243. doi:10.1016/S0012-365X(97)00143-X

[13] L. Milazzo, Zs. Tuza and V. Voloshin, Strict coloring of triple and quadruple Steiner systems: a survey, Discrete Math. 261 (2003) 399-411. doi:10.1016/S0012-365X(02)00485-5

[14] L. Milazzo and Zs. Tuza, Logarithmic upper bound for upper chromatic number of $S(t, t+1, v)$ systems, Ars Combin. 92 (2009) 213-223.

[15] I. Tomescu, Chromatic coefficients of linear uniform hypergraphs, J. Combin. Theory (B) 72 (1998) 229-235. doi:10.1006/jctb.1997.1811

[16] V.I. Voloshin, The mixed hypergraphs, Comput. Sci. J. Moldova 1 (1993) 45-52.

[17] V.I. Voloshin, On the upper chromatic number of a hypergraph, Australas. J. Combin. 11 (1995) 25-45.

[18] V.I. Voloshin, Coloring Mixed Hypergraphs: Theory, Algorithms and Applications (American Mathematical Society, Providence 2002).

[19] M. Walter, Some results on chromatic polynomials of hypergraphs, Electron. J. Combin. 16 (2009) \#R94.

Received 9 January 2013

Revised 13 June 2013

Accepted 13 June 2013 